\title{
Prediction of the critical thermal zone during pulmonary cryoablation on computed tomography from correlated experimental and clinical findings
}

\author{
Kohei Hashimoto, MD, ${ }^{\mathrm{a}}$ Yotaro Izumi, MD, PhD, ${ }^{\mathrm{a}}$ Yoshikane Yamauchi, MD, ${ }^{\mathrm{a}}$ Hideki Yashiro, MD, \\ Masanori Inoue, MD, ${ }^{\mathrm{b}}$ Seishi Nakatsuka, MD, PhD, ${ }^{\mathrm{b}}$ and Hiroaki Nomori, $\mathrm{MD}, \mathrm{PhD}^{\mathrm{a}}$
}

\begin{abstract}
Objective: During cryoablation, cells are destroyed at temperatures less than $-20^{\circ} \mathrm{C}$. The determining factors for local cancer control in pulmonary cryoablation were assessed using computed tomography (CT), isothermal curves, and histologic findings in pigs. Experimental findings were compared with clinical CT findings and were extrapolated to local cancer control outcomes.
\end{abstract}

\begin{abstract}
Methods: Cryoablation was performed with thermal monitoring, and the ablated areas were divided into 3 zones: less than $-20^{\circ} \mathrm{C},-20^{\circ} \mathrm{C}$ to $0^{\circ} \mathrm{C}$, and greater than $0^{\circ} \mathrm{C}$ and were compared with histologic findings. CT findings with multiplanar reconstruction in 36 nodules were compared with the porcine histologic findings. The relationship between CT findings and 3-year local cancer control was evaluated in 98 nodules.

Results: The 3 concentric thermal zones correlated with histologic findings as follows: less than $-20^{\circ} \mathrm{C}$ zone, complete tissue destruction (zone D); $-20^{\circ} \mathrm{C}$ to $0^{\circ} \mathrm{C}$ zone (which surrounded zone D), hemorrhage with air trapping and maintenance of alveolar structures (zone $\mathrm{H}$ ); and greater than $0^{\circ} \mathrm{C}$ zone (outermost), edema with sustained alveolar structures (zone E). The CT findings in 36 nodules showed a central solid zone, a surrounding air-containing zone, and an outside solid zone, corresponding to zones $\mathrm{D}, \mathrm{H}$, and $\mathrm{E}$, respectively. Local cancer control at 3 years in 80 nodules contained within the central solid zone was significantly greater compared with the 18 nodules that were not $(82 \%$ vs $33 \%, P=.0002)$.
\end{abstract}

Conclusions: Pulmonary cryoablation should be performed such that tumors are contained within the central solid zone on CT, which represents the less than $-20^{\circ} \mathrm{C}$ zone. (J Thorac Cardiovasc Surg 2013;145:832-8)

Recent advances in cryoablation devices have contributed to the development of minimally invasive options for local control of cancer in various organs. ${ }^{1-4}$ For lung tumors, percutaneous cryoablation was first reported by Wang and colleagues ${ }^{4}$ in 2005 . Percutaneous cryoablation for lung tumors has been performed at our institution since 2002 and has proved both feasible and safe. ${ }^{5}$ Improvement in local control is the next important issue. Although evidence has suggested that a temperature of $-20^{\circ} \mathrm{C}$ or lower must be reached to destroy cells in solid tumors organs, ${ }^{6-12}$ the thermal conductivity of the lung has been reported to be different from that of solid organs because it contains air. ${ }^{13,14}$ Therefore, the extent of the zone of tissue that reaches temperatures less than $-20^{\circ} \mathrm{C}$ after pulmonary cryoablation is still not well established. In the present

\footnotetext{
From the Division of General Thoracic Surgery, ${ }^{\mathrm{a}}$ Department of Surgery, and Department of Diagnostic Radiology, ${ }^{b}$ Keio University School of Medicine, Tokyo, Japan.

Disclosures: Authors have nothing to disclose with regard to commercial support. Received for publication Nov 26, 2011; revisions received March 4, 2012; accepted for publication March 16, 2012; available ahead of print April 16, 2012. Address for reprints: Hiroaki Nomori, MD, PhD, Department of General Thoracic Surgery, Keio University School of Medicine, 35 Shinanomachi, Shinjuku-ku, Tokyo 160-8582, Japan (E-mail: hnomori@qk9.so-net.ne.jp). $0022-5223 / \$ 36.00$

Copyright (c) 2013 by The American Association for Thoracic Surgery doi:10.1016/j.jtcvs.2012.03.029
}

study, using a custom-designed 2-dimensional thermocouple device, ${ }^{15}$ cryoablation of the lung was performed in pigs, and the correlation between the isothermal curves and histologic findings was examined. These findings were also compared with the clinical findings on computed tomography (CT).

\section{METHODS}

\section{Animal Experiments}

The experiment was performed using male domestic pigs ( 3 months old, approximate weight $40 \mathrm{~kg}, \mathrm{n}=12$ ). Under general anesthesia, the pigs were mechanically ventilated at a respiratory rate of 15 to 20 breaths/min with a tidal volume of $10 \mathrm{~mL} / \mathrm{kg}$. Each pig was placed in the left decubitus position, and right thoracotomy was performed. The posterior lobe of the right lung was used, because it has the largest volume; therefore, the possibility of a large vessel existing in the vicinity of the probe and affecting the experiment was considered to be lowest in this lobe. Cryoablation was performed using a 2.4-mm cryoprobe (CRYO-care Cryosurgical Unit; Endocare, Irvine, Calif) in 2 locations more than $5 \mathrm{~cm}$ apart, in each pig. Type K thermocouples were used for temperature measurement, as previously described. ${ }^{15}$ Temperature measurements were made simultaneously at 40 points every 5 seconds using a data logger (Pico Technology, Cambridgeshire, UK). A 2.4-mm cryoprobe was inserted at the center, and 4 thermocouples each at 4-, 6-, 8-, and 10-mm positions from the center and 8 thermocouples each at 13-, 15-, and 17-mm positions from the center were fixed using an acrylic device (Yokohama Micro Giko, Yokohama, Japan; Figure 1,A). Preliminary experiments showed that the largest radius of a ball of ice generated in egg white was approximately $10 \mathrm{~mm}$, measured from the tip of the cryoprobe. Therefore, to assess the maximum freezing 


\section{Abbreviations and Acronyms \\ $\mathrm{CI}=$ confidence interval \\ $\mathrm{CT}=$ computed tomography \\ $\mathrm{ISO}=$ isothermal \\ $\mathrm{HIS}=$ histologic}

capacity of the cryoprobe, the temperature change was measured with the thermocouples positioned $8 \mathrm{~mm}$ from the acrylic plate and the cryoprobe fixed $18 \mathrm{~mm}$ from the acrylic plate (Figure 1,B). Figure 1, $C$ illustrates cryoprobe insertion and temperature measurement. The freezing and thawing by the cryoprobe is based on the Joule-Thomson effect of the rapid expansion of high-pressure argon, for freezing, and helium, for thawing, respectively. The tip of the cryoprobe reaches approximately $-130^{\circ} \mathrm{C}$ during freezing and approximately $40^{\circ} \mathrm{C}$ during thawing. Three freeze-thaw cycles were conducted, as previously described. ${ }^{15}$ This is the same protocol as that used in the clinical setting at our institution. For the freeze-thaw cycle, the first freezing time was 5 minutes, and the second and third freezing periods were 10 minutes each. For the first and second thaw periods, active thawing was performed until the temperature reached $20^{\circ} \mathrm{C}$. The helium gas was then stopped, and passive thawing was allowed until the total thawing time reached 10 minutes. For the third thaw period, high-pressure helium gas was used until the cryoprobe was removed. The lungs were resected immediately after cryoablation and were fixed using bronchial injection of $10 \%$ formalin at $20 \mathrm{~cm} \mathrm{H}_{2} \mathrm{O}$. All animal studies were approved by the Keio University School of Medicine, Institutional Animal Care and Use Committee and were performed in accordance with the "Guide for the Care and Use of Laboratory Animals" published by the National Institutes of Health (Bethesda, Md).

\section{Histologic Examination}

Fixed specimens were cut to represent the planes of the isothermal curves (ie, the specimens were cut perpendicular to the probe insertion route, approximately $8 \mathrm{~mm}$ from the lung surface). The slides were prepared by cutting each specimen into quarters with the point of cryoprobe insertion at the center. The ablated region was evaluated using $4-\mu \mathrm{m}$ sections stained with hematoxylin and eosin.

\section{Pulmonary Cryoablation in Clinical Practice}

Most patients in the present study had metastatic lung tumors and were considered unsuitable for surgical resection owing to comorbidities or oncologic considerations. The disease extent was evaluated primarily by CT. Positron emission tomography was used for some patients. Pulmonary cryoablation was conducted in the patients, as previously reported. ${ }^{5}$ In brief, under a multidetector-row CT scanner with multislice CT fluoroscopy functions (Aquilion 64; Toshiba Medical, Tokyo, Japan), using an outer insertion sheath, a 1.7-mm-diameter cryoprobe (CRYO-care Cryosurgical Unit; Endocare) was inserted into a targeted nodule under fluoroscopic CT guidance. The cryoprobe used high-pressure argon and helium gas for freezing and thawing, respectively, according to the Joule-Thompson principle. Cryoablation consisted of 3 cycles of freezing for 5,10 , and 10 minutes each. The tip of the cryoprobe reaches approximately $-130^{\circ} \mathrm{C}$ during freezing. The protocol of the 3 freeze-thaw cycles has been previously described in the animal experiments. The institutional review board approved the study, and all patients provided written informed consent.

\section{Clinical CT Image Analysis}

High-resolution CT (HRCT) images taken immediately after cryoablation were retrospectively reviewed in patients from 2006 to 2011 for comparison with the experimental data. From 2006 to 2011, 302 tumors in 118 patients were treated with cryoablation. Of these, 118 tumors in 64 patients treated with 1 probe per tumor were selected for comparison of the CT findings with the experimental data using 1 probe. Of the 118 tumors, 82 were excluded because of emphysematous or interstitial changes in the surrounding lung parenchyma that could interfere with image analysis. The HRCT images of the remaining 36 tumors were examined. The images were reconstructed perpendicular to the cryoprobe, approximately 10 $\mathrm{mm}$ from the tip, to be comparable with the experimental data.

To evaluate the long-term local cancer control after cryoablation, the HRCT images of 98 tumors in 57 patients treated from 2002 to 2006 were examined. All patients were treated using a single cryoprobe per tumor. The tumors were grouped according to whether the tumor was completely contained within zone D on CT (CT-zone D) (contained group) or not contained within zone D (noncontained group). CT-zone D was verified from axial slices in these 2 groups. The local control rates were compared between the 2 groups. Only local control was assessed for patients treated from 2002 to 2006 because of practical considerations. First, since 2006 , all the tumors treated with cryoablation have been intentionally contained within CT-zone D. The inclusion of these treatment outcomes would not allow proper comparisons and could have biased the results. Second, the HRCT images obtained from 2002 to 2006 could not be reconstructed perpendicular to the cryoprobe for image analysis, because these data were stored as films. The institutional review board approved the present retrospective study protocol.

\section{Follow-up After Cryoablation}

All patients were followed up using CT imaging every 3 to 6 months after cryoablation. Typically, the lesion increased in size after cryoablation and then sequentially regressed over a period of several months. Most lesions form scars, which can vary in shape and size. Local progression was confirmed when diffuse enlargement of the scar lesion was seen and the proportional increase in the longest diameter exceeded $20 \%$ during follow-up, according to the Response Evaluation Criteria in Solid Tumors. Local progression was also confirmed when focal enlargement was present, typically focal thickening of the scar, and the proportional increase in the longest diameter of the focal thickening exceeded $20 \%$. These changes were evaluated over a period of at least 2 months. Otherwise, the lesions were defined as locally controlled. We also evaluated locoregional progression, which was confirmed by local recurrence and/or the appearance of pulmonary metastases in the same lobe and/or the appearance of ipsilateral lymph node metastasis.

\section{Statistical Analysis}

The equivalence in mean proportions estimated using 3 modalities (ie, isothermal curves, histologic findings, and clinical CT scans) was tested by comparing the $95 \%$ confidence intervals (CIs) for the difference in mean proportions with a margin of $\pm 10 \%$. The correlation between groups was evaluated using Pearson's $r$ test. The local control curves in the 2 groups were estimated with the Kaplan-Meier method and were compared using the log-rank test. The significance level for all tests was 2-sided, at $5 \%$. A comparison of values between groups was done using the unpaired $t$ test. All data were analyzed using IBM SPSS Statistics, version 19, software (IBM, Armonk, NY). The data are presented as mean \pm standard deviation.

\section{RESULTS \\ Isothermal Curves}

Using the thermocouples, 2-dimensional concentric isothermal curves were constructed for each cryoablation $(\mathrm{n}=24$; Figure 2$)$. The ablated area was divided into 3 zones: less than $-20^{\circ} \mathrm{C}$ zone (isothermal [ISO]-zone D), $-20^{\circ} \mathrm{C}$ to $0^{\circ} \mathrm{C}$ zone (ISO-zone $\mathrm{H}$ ), and greater than $0^{\circ} \mathrm{C}$ zone (ISO-zone E). The average area of the ISO-zone 

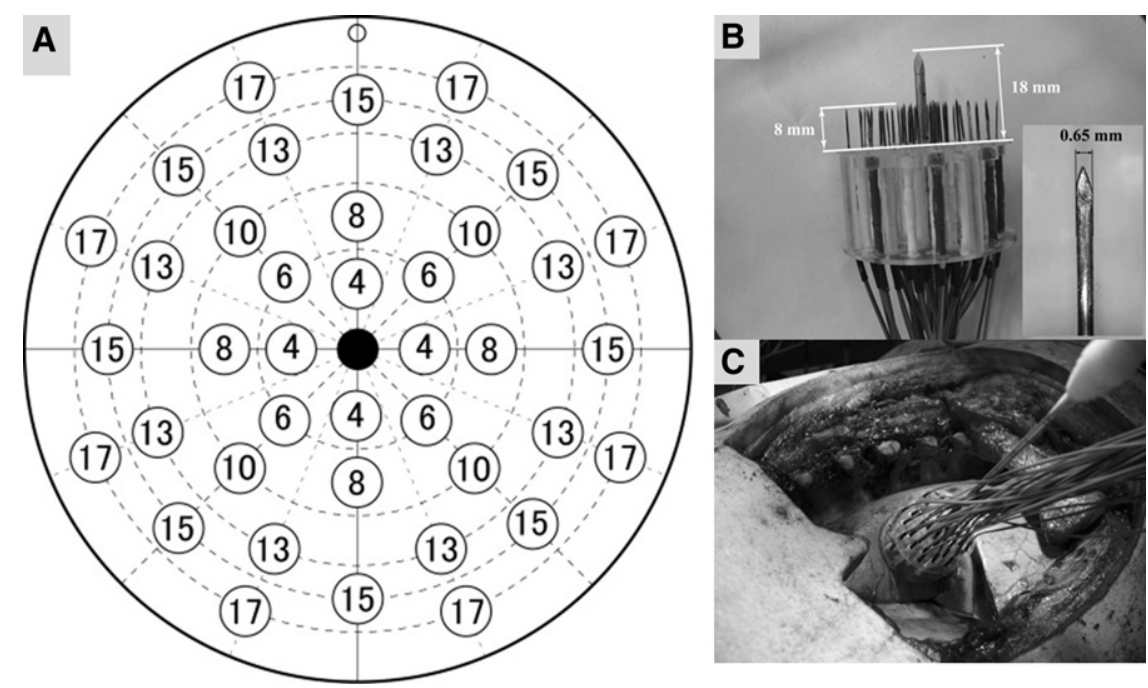

FIGURE 1. A, Position of the cryoprobe at the center and thermocouples. The numbers indicate the distances ( $\mathrm{mm}$ ) from the center. B, The cryoprobe and measurement device. The cryoprobe at the center and thermocouples are fixed at $18 \mathrm{~mm}$ and $8 \mathrm{~mm}$ from the tip to the acrylic plate, respectively. The tip of a thermocouple is shown in the inset. C, Image demonstrating how the temperature is measured. Cryoprobe and thermocouples are inserted to the posterior lobe of the pig lung.

D was $258 \mathrm{~mm}^{2} \pm 47 \mathrm{~mm}^{2}$, and the average area of the ISOzone $\mathrm{D}+\mathrm{H}$ was $448 \mathrm{~mm}^{2} \pm 78 \mathrm{~mm}^{2}$. The average radius of the ISO-zone $\mathrm{D}$ was $9 \mathrm{~mm} \pm 4 \mathrm{~mm}$ and that of the ISO-zone $\mathrm{D}+\mathrm{H}$ was $12 \mathrm{~mm} \pm 5 \mathrm{~mm}$.

\section{Histologic Findings}

From the histologic specimens, 3 concentric regions were observed around the probe insertion site (Figure 3, A). A complete destruction zone (HIS-zone D) in the most central region was identified, along with a hemorrhagic zone
(HIS-zone $\mathrm{H})$ in the outer region and an edema zone (HIS-zone E) in the outermost region. In HIS-zone D, the alveolar structures were no longer apparent, and both severe hemorrhage and edema were present (Figure 3, B). In HISzone $\mathrm{H}$, to the outside of HIS-zone $\mathrm{D}$, severe hemorrhage was seen, although alveolar structures with trapped air remained (Figure 3, C). In HIS-zone E, outside of HIS-zone $\mathrm{H}$, extensive pulmonary edema with preserved alveolar structures was seen, but air trapping was not present (Figure 3, D).

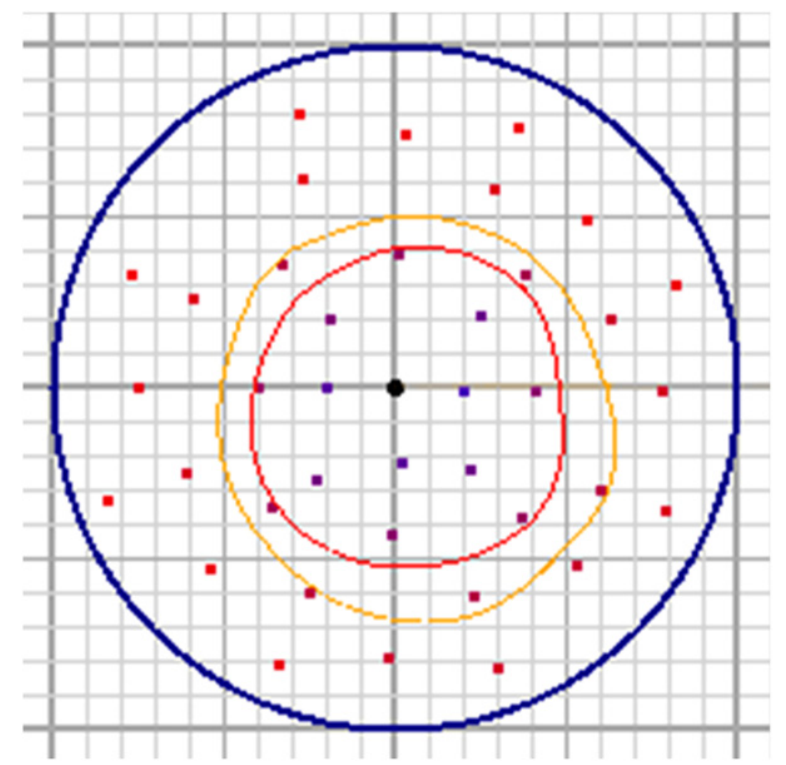

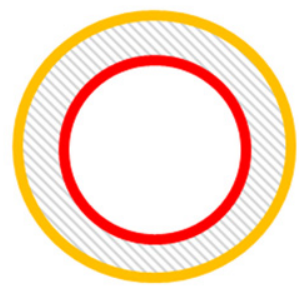

\section{-20 to $0{ }^{\circ} \mathrm{C}$ zone} (ISO-zone H)

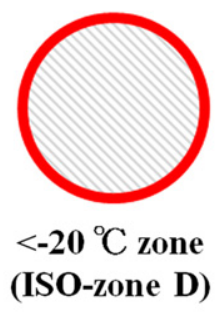

FIGURE 2. A representative isothermal curve. The less than $-20^{\circ} \mathrm{C}$ zone (isothermal [ISO]-zone D) is illustrated within the red inner circle. The $-20^{\circ} \mathrm{C}$ to $0^{\circ} \mathrm{C}$ (ISO-zone $\mathrm{H}$ ) zone is illustrated within the yellow outer circle. The outermost blue line represents the outer edge of the acrylic plate. 


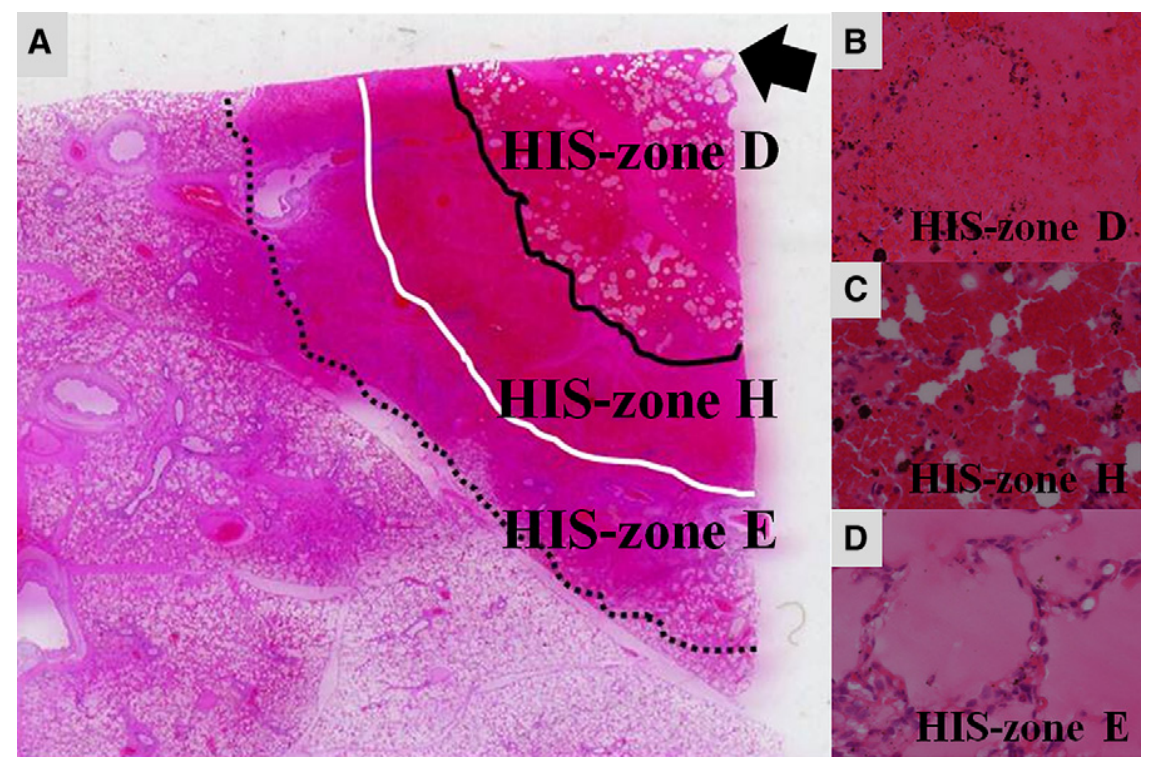

FIGURE 3. A, A representative histologic specimen obtained by cryoablation. Three concentric regions (histologic [HIS]-zone D, H, and E) could be identified around the probe insertion site (arrow). Specimen sectioned perpendicular to the probe. B, HIS-zone D showed severe hemorrhage and edema and the alveolar structures were no longer apparent. C, HIS-zone $\mathrm{H}$ showed severe pulmonary hemorrhage. The alveolar structures could be identified but were not distinct. There were also findings of trapped air within these structures. D, HIS-zone E showed extensive pulmonary edema. The alveolar structures were maintained. B-D, Hematoxylin and eosin staining, original magnification $\times 40$.

\section{Relation of Thermal Zones and Histologic Findings}

The average area ratio of the ISO-zone D/ISO-zone D+H was $58 \% \pm 2 \%$, almost identical to that of HIS-zone D/HIS-zone $\mathrm{D}+\mathrm{H}(57 \% \pm 1 \%)$. The $95 \% \mathrm{CI}$ for the difference in mean proportions demonstrated statistical equivalence between these 2 ratios $(95 \%$ CI for difference, $-1.6 \%$ to $+0.4 \%)$. Also, the areas of ISO-zone D and ISO-zone $\mathrm{D}+\mathrm{H}$ correlated significantly with the areas of HIS-zone D and HIS-zone D $+\mathrm{H}$, respectively (Pearson's correlation coefficient $\mathrm{r}=0.98$ and 0.96 , respectively, and $P<.001$ and $P<.001$, respectively, Pearson's $r$ test). These results suggest that HIS-zone D corresponds to the ISOzone $\mathrm{D}$ region and that a temperature of less than $-20^{\circ} \mathrm{C}$ was responsible for the extensive tissue destruction seen in HIS-zone D. The results also suggest that the area of the less than $-20^{\circ} \mathrm{C}$ zone represented approximately $57 \%$ of the macroscopic ice ball, the border of which represents a temperature of $0^{\circ} \mathrm{C}$.

\section{Clinical CT Image Analysis}

The tumor characteristics of the 36 nodules used for image analysis were as follows. The tumor size ranged from 9 $\mathrm{mm}$ to $20 \mathrm{~mm}$ (mean, $12 \mathrm{~mm} \pm 4 \mathrm{~mm}$ ); 7 cases were primary nonsmall-cell lung carcinoma, and 29 cases were metastatic lung tumors from nonsmall-cell lung carcinoma in 6, colorectal cancer in 5, laryngeal cancer in 3, leiomyosarcoma in 2, salivary gland carcinoma in 2 , renal cell carcinoma in 2, esophageal cancer in 1 , neck cancer in 1 , chondrosarcoma in 1, prostate cancer in 1, ovarian cancer in 1, osteosarcoma in 1, papillary thyroid carcinoma in 1 , breast cancer in 1, and gingival sarcoma in 1. The HRCT images in all 36 nodules showed 3 concentric regions around the cryoprobe insertion site as follows: CT-zone $\mathrm{D}, \mathrm{CT}$-zone $\mathrm{H}$, and CT-zone $\mathrm{E}$ (Figure $4, A-C$ ). The average area of the CT-zone D was $193 \mathrm{~mm}^{2} \pm 69 \mathrm{~mm}^{2}$ and that of the CT-zone $\mathrm{D}+\mathrm{H}$ was $368 \mathrm{~mm}^{2} \pm 116 \mathrm{~mm}^{2}$, both of which were smaller than the corresponding experimental areas of HIS-zone D $\left(258 \mathrm{~mm}^{2} \pm 47 \mathrm{~mm}^{2}\right)$ or HIS-zone D+H (448 $\mathrm{mm}^{2} \pm 78 \mathrm{~mm}^{2}$ ). However, the ratio of the areas, CTzone D/CT-zone $\mathrm{D}+\mathrm{H}$ was $52 \% \pm 11 \%$, which was similar to the experimental data $(57 \% \pm 1 \%)$. The average area ratios for CT-zone D/CT-zone D+H, ISO-zone D/ISO-zone $\mathrm{D}+\mathrm{H}$, and HIS-zone D/HIS-zone $\mathrm{D}+\mathrm{H}$ were all statistically equivalent (CT vs thermal zones, 95\% CI for difference, $-8.9 \%$ to $-1.3 \%$; histology vs CT, $95 \%$ CI for difference, $-8.2 \%$ to $-0.8 \%$ ). These results suggest that the CT-zone $\mathrm{D}$ also corresponds to the less than $-20^{\circ} \mathrm{C}$ zone.

\section{Comparison of Local Control Rates From Clinical CT Data}

The clinical characteristics of the 98 nodules used for comparison of local control rates are listed in Table 1 . The average nodule size was not significantly different between the contained group and noncontained group $(P=.17$, unpaired $t$ test). The 3-year local control rate in the contained group was significantly greater than that in the noncontained group ( $82 \%$ vs $33 \%$; Figure 5, A). Qualitatively, if we regarded the dramatic decrease in size as 


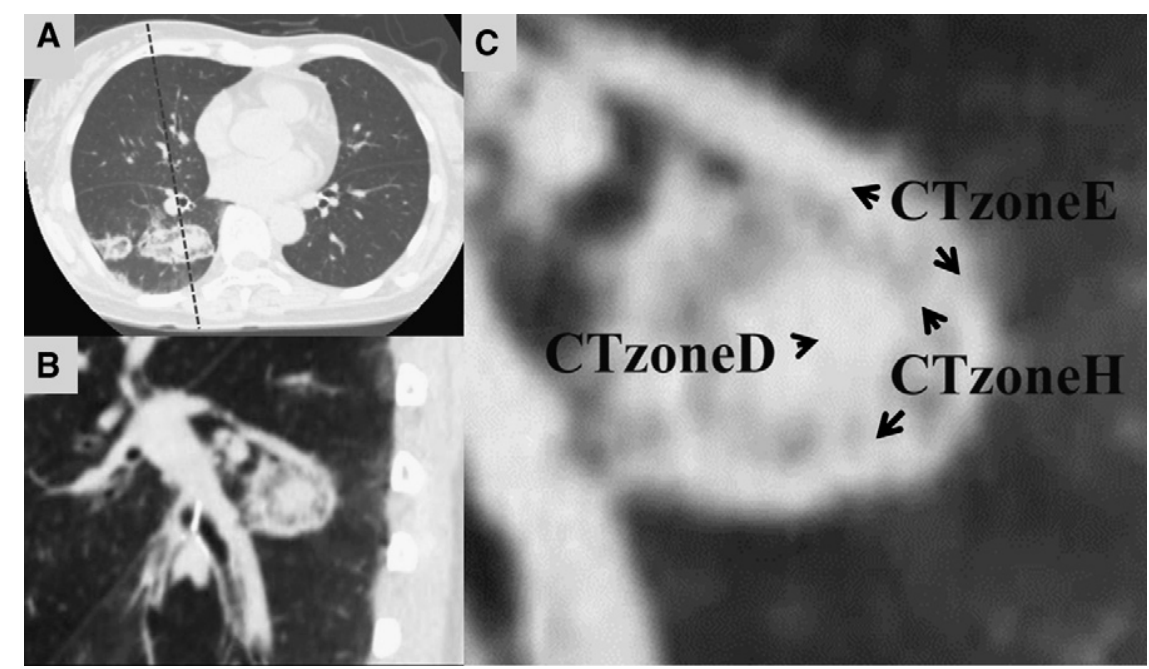

FIGURE 4. A, A representative clinical computed tomography $(C T)$ scan immediately after cryoablation. Multiplanar reconstruction was performed along the plane (dotted line), which ran perpendicular to the tract of the cryoprobe and was $10 \mathrm{~mm}$ proximal from the estimated tip of the cryoprobe. B, Three concentric regions around the cryoprobe insertion site, similar to the histologic findings, could be identified. These regions were arbitrarily named CT-zone D, CT-zone $\mathrm{H}$, and CT-zone E.

formations of very thin or faint scars, 11 patients in the contained group (14\%) and 4 patients in the noncontained group $(22 \%)$ had dramatic deceases in size at some point during follow-up. The 3-year locoregional control rate in the contained group tended to be greater than that in the

TABLE 1. Characteristics of 98 nodules examined for local control outcome after cryoablation

\begin{tabular}{lcc}
\hline \multicolumn{1}{c}{ Characteristic } & $\begin{array}{c}\text { Noncontained } \\
\text { group* }\end{array}$ & $\begin{array}{c}\text { Contained } \\
\text { group } \dagger\end{array}$ \\
\hline Nodule size (mm) & $15 \pm 3 \ddagger$ & $12 \pm 3 \ddagger$ \\
Mean & $9-20$ & $8-20$ \\
Range & & \\
Histologic type & 2 & 3 \\
Primary NSCLC & & \\
Metastatic lung cancer & 4 & 34 \\
NSCLC & 5 & 12 \\
Colorectal cancer & 2 & 3 \\
Leiomyosarcoma & 0 & 4 \\
Salivary gland carcinoma & 0 & 9 \\
Renal cell carcinoma & 1 & 1 \\
Neck cancer & 2 & 2 \\
Uterine cervical cancer & 1 & 0 \\
Pancreatic cancer & 1 & 0 \\
Uterine cancer & 0 & 5 \\
Hepatocellular carcinoma & 0 & 3 \\
Synovial sarcoma & 0 & 2 \\
MFH & 0 & 1 \\
MPNST & 0 & 80 \\
Gingival sarcoma & 18 & 1 \\
Total & & \\
\hline
\end{tabular}

NSCLC, Non-small cell lung carcinoma; $M F H$, malignant fibrous histiocytoma; MPNST, malignant peripheral nerve sheath tumor. *Nodules not contained within central solid zone (CT-zone D). †Nodules contained within central solid zone (CT-zone D). $\ddagger P=.17$, unpaired $t$ test. noncontained group, but this difference was not statistically significant ( $61 \%$ vs $33 \%$; Figure $5, B)$. Of the patients with locoregional recurrence, $52 \%$ also had concurrent extralocoregional recurrences.

\section{DISCUSSION}

The present study highlights the following findings. The less than $-20^{\circ} \mathrm{C}$ thermal zone (ISO-zone D), which was the most central zone surrounding the cryoprobe, corresponded to the zone of complete destruction (HIS-zone D) on histologic analysis. The less than $-20^{\circ} \mathrm{C}$ thermal zone also corresponded to the central solid zone on CT (CT-zone D). Finally, the CT image analysis suggested that nodules contained within the central solid zone (CT-zone D) had significantly greater local cancer control rates.

The CT-zone D and CT-zone D $+\mathrm{H}$ were smaller than experimental zones $\mathrm{D}$ and $\mathrm{D}+\mathrm{H}$, respectively. This is likely because the CT measurements were taken in the deeper regions of the lung, which were warmer owing to the increased relative blood flow, compared with measurements made in more peripheral lung regions in the animal experiments. However, the area ratio of CT-zone D/CT-zone $\mathrm{D}+\mathrm{H}$ was similar to that of experimental zone $\mathrm{D} /$ zone $\mathrm{D}+\mathrm{H}$. This suggests that these regions, as reproduced in the animal experiments, could represent the 3 concentric regions observed clinically on the CT images.

In the present study, the radius of the ISO-zone D was approximately $9 \mathrm{~mm}$. This was slightly smaller than that reported in a previous study by Hinshaw and colleagues, ${ }^{16}$ in which the diameter of the less than $-20^{\circ} \mathrm{C}$ region was $23 \mathrm{~mm}$. This difference could be attributable to the subtle differences in the capacity of the probes and/or the system used. The difference could also be explained because the 


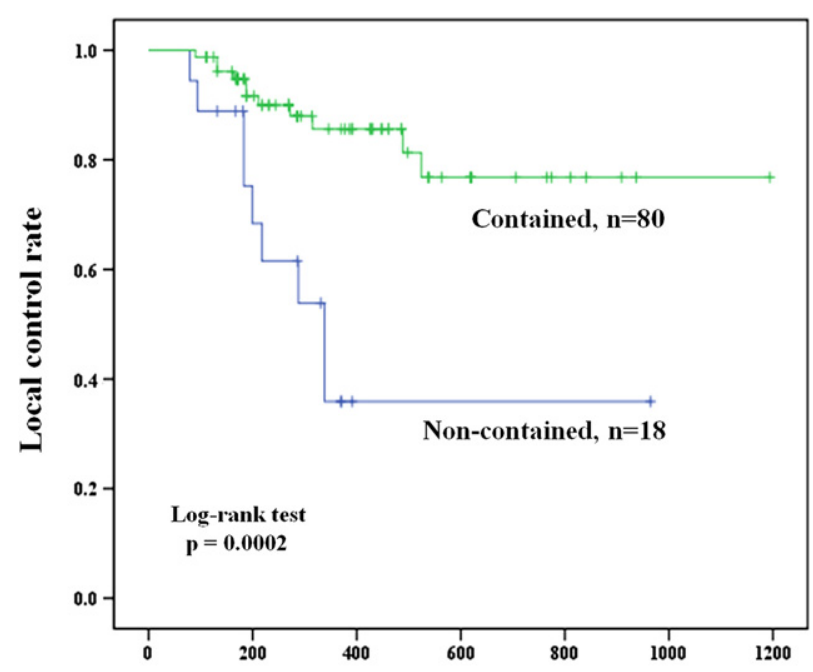

A

Time in days

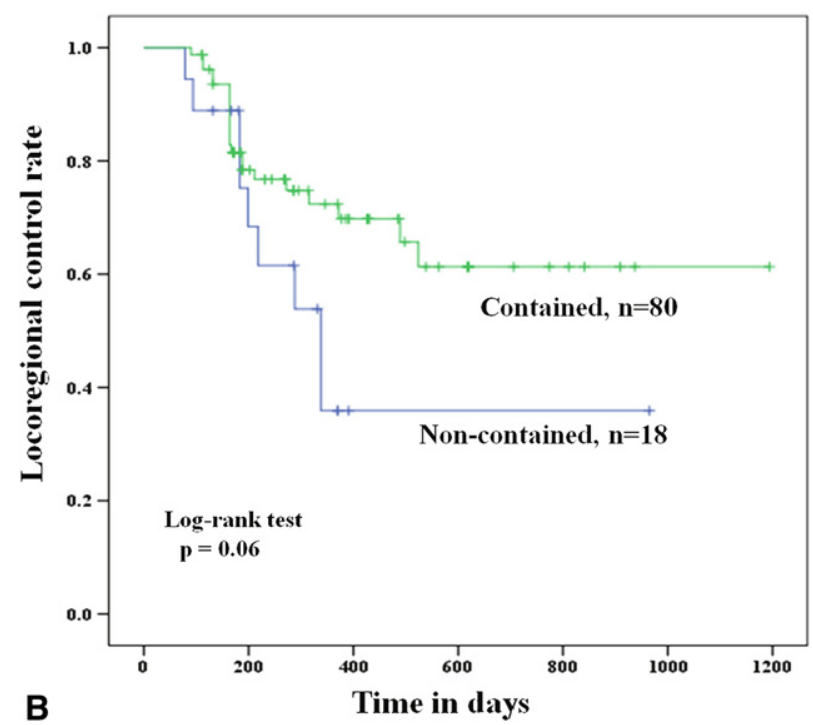

FIGURE 5. A, The local cancer control rates in the contained and noncontained groups. The local tumor control rate was significantly decreased when the tumor was not contained within computed tomography (CT)zone D (noncontained group) compared with full tumor containment within CT-zone D (contained group; 3-year local control rate, 34\% vs $82 \% ; P=.0002, \log$-rank test). B, The locoregional cancer control rates were not significantly different when the tumor was not contained within CT-zone D (noncontained group) compared with full tumor containment within CT-zone D (contained group; 3-year local control rate, 33\% vs $61 \% ; P=.06$, log-rank test).

thermocouple measurement was made in only 1 direction in their study. However, the ratio of the less than $-20^{\circ} \mathrm{C} / 20$ to $0{ }^{\circ} \mathrm{C}$ area reported by Hinshaw and colleagues,${ }^{16}$ was $54 \%$, similar to the values obtained in the present study.

The results of the present study must be considered in light of the following limitations. First, the thermal curve data and histologic findings were matched according to the comparative values (ie, ratio of zone areas), rather than the absolute values. However, currently, no effective method is available to accurately represent the location of the thermocouples on histologic findings, which would potentially address this limitation. Second, the thermal conductivity might differ between solid tumor tissue and air-containing lung tissue. This can only be addressed by incorporating a thermocouple device into clinical practice. Although it might be possible to insert a thermocouple device through the chest wall under fluoroscopic CT guidance, 3-dimensional CT image reconstruction would be required to appropriately adjust the distance between the probe and the thermocouples. This process would be too time consuming in clinical practice.

In the clinical data, we consider that the difference in the containment of the tumor in the CT-zone D related primarily to the suboptimal placement of the probe. Although not statistically significant, the noncontained group had larger tumors (15 mm vs $12 \mathrm{~mm}$ ), and this might also, at least in part, have been the cause of insufficient tumor containment and the decreased local control rate. Also, the clinical data showed that the local cancer control rate was not $100 \%$, even if the tumor was fully contained within CT-zone D. The data could have been affected by alterations in thermal conductivity by the existence of tumor tissue compared with the experimental settings in which no tumor was present. Therefore, the data from the pig lungs might not be directly applicable to the clinical findings. Ablate and resect studies, ${ }^{17}$ if considered feasible, might provide some answers to this issue. Furthermore, the condition of lung tissue is likely more variable among patients than in controlled animal experiments. Additional data from clinical studies are necessary to address these issues.

Most patients in the present study were being treated for metastatic lesions; therefore, a distinction between local recurrence and the appearance of new lesions was required. However, at least in the present study, when sequential CT images were examined, we found that it was not difficult to distinguish local recurrence from the appearance of a new lesion in the proximity of the ablated lesion scar.

Locoregional control tended to be better in the contained group than in the noncontained group; however, the difference was not statistically significant. Because $52 \%$ of the patients with locoregional recurrence also had concurrent distant metastases and/or pulmonary metastases in other lobes, we believe that locoregional control was difficult to evaluate in the present study group composed primarily of patients with metastatic lung tumors.

The present study has demonstrated that the less than $-20^{\circ} \mathrm{C}$ zone corresponds to the central solid zone on CT. Local cancer control was better in nodules contained within this zone. Therefore, pulmonary cryoablation should target tumors contained within the central solid zone on $\mathrm{CT}$, which represents the less than $-20^{\circ} \mathrm{C}$ zone. 
We thank Drs Takayuki Abe and Yuji Sato, Center for Clinical Research, Keio University School of Medicine, Tokyo, Japan, for their valuable help in the statistical analysis. We also thank Drs Taisuke Nagasawa and Kansei Iwata, DGS Computer Company, Ltd, Tokyo, Japan, for their assistance in the isothermal curve data acquisition and analysis.

\section{References}

1. Bahn DK, Lee F, Badalament R, Kumar A, Greski J, Chernick M. Targeted cryoablation of the prostate: 7-year outcomes in the primary treatment of prostate cancer. Urology. 2002;60:16-20.

2. Korpan NN. Hepatic cryosurgery for liver metastases: long-term follow-up. Ann Surg. 1997;225:193-201.

3. Gill IS, Novick AC, Soble JJ, Sung GT, Remer EM, Hale J, et al. Laparoscopic renal cryoablation: initial clinical series. Urology. 1998;52:543-51.

4. Wang H, Littrup PJ, Duan Y, Zhang Y, Feng H, Nie Z. Thoracic masses treated with percutaneous cryotherapy: initial experience with more than 200 procedures. Radiology. 2005;235:289-98.

5. Kawamura M, Izumi Y, Tsukada N, Asakura K, Sugiura H, Yashiro H, et al. Percutaneous cryoablation of small pulmonary malignant tumors under computed tomographic guidance with local anesthesia for nonsurgical candidates. J Thoracic Cardiovasc Surg. 2006;131:1007-13.

6. Campbell SC, Krishnamurthi V, Chow G, Hale J, Myles J, Novick AC. Renal cryosurgery: experimental evaluation of treatment parameters. Urology. 1998; 52:29-33.
7. Rupp CC, Hoffmann NE, Schmidlin FR, Swanlund DJ, Bischof JC, Coad JE. Cryosurgical changes in the porcine kidney: histologic analysis with thermal history correlation. Cryobiology. 2002;45:167-82.

8. Gage AA, Baust JM, Baust JG. Experimental cryosurgery investigations in vivo. Cryobiology. 2009;59:229-43.

9. Gage AA, Baust JM. Mechanisms of tissue injury in cryosurgery. Cryobiology. 1998;37:171-86.

10. Jacob G, Kurzer MN, Fuller BJ. An assessment of tumor cell viability after in vitro freezing. Cryobiology. 1985;22:417-26.

11. McGrath JJ, Cravalho EG, Huggins CE. An experimental comparison of intracellular ice formation and freeze-thaw survival of HeLa S-3 cells. Cryobiology. 1975;12:540-50.

12. Tatsutani K, Rubinsky B, Onik G, Dahiya R. Effect of thermal variables on frozen human primary prostatic adenocarcinoma cells. Urology. 1996;48:441-7.

13. Izumi Y, Oyama T, Ikeda E, Kawamura M, Kobayashi K. The acute effects of transthoracic cryoablation on normal lung evaluated in a porcine model. Ann Thoracic Surg. 2005;79:318-22.

14. Lee CY, Bastacky J. Comparative mathematical analyses of freezing in lung and solid tissue. Cryobiology. 1995;32:299-305.

15. Nakatsuka S, Yashiro H, Inoue M, Kuribayashi S, Kawamura M, Izumi Y, et al. On freeze-thaw sequence of vital organ of assuming the cryoablation for malignant lung tumors by using cryoprobe as heat source. Cryobiology. 2010;61:317-26.

16. Hinshaw JL, Sampson L, Lee FT Jr, Laeseke PF, Brace CL. Does selective intubation increase ablation zone size during pulmonary cryoablation? J Vasc Interv Radiol. 2008;19:1497-501.

17. Schneider T, Reuss D, Warth A, Schnabel PA, von Deimling A, Herth FJF, et al. The efficacy of bipolar and multipolar radiofrequency ablation of lung neoplasmsresults of an ablate and resect study. Eur J Cardiothorac Surg. 2011;39:968-73. 